Acta Sci. Pol. Technol. Aliment. 20(3) 2021, 313-323

pISSN 1644-0730

eISSN 1898-9594

http://dx.doi.org/10.17306/J.AFS.2021.0943

ORIGINAL PAPER

Received: 9.03.2021

Accepted: 14.05.2021

\title{
THE REGULATION OF 1-METHYLCYCLOPROPENE TREATMENT ON THE SUBFAMILY GENES EXPRESSION OF ETHYLENE RESPONSE FACTORS IN PEACHES DURING STORAGE
}

\author{
Hongfang Cai ${ }^{1}$, Shuai Han², Hui Wang ${ }^{1}$, Mingliang Yu³, Ruijuan $\mathrm{Ma}^{3}$, Zhifang $\mathrm{Yu}^{4 \bowtie}$ \\ ${ }^{1}$ Changshu Institute of Technology \\ South Third Ring 99, 215500 Changshu, PR China \\ ${ }^{2}$ College of Food and Drug, Shanghai Zhongqiao Vocational and Technical University \\ Caolang 3888, 201514 Shanghai, PR China \\ ${ }^{3}$ Institute of Pomology, Jiangsu Academy of Agricultural Sciences, Jiangsu Key Laboratory for Horticultural Crop Genetic \\ Improvement, Zhongling 50, Nanjing, 210014 Jiangsu, PR China \\ ${ }^{4}$ College of Food Science and Technology, Nanjing Agricultural University \\ Weigang 1, Nanjing, 210095 Jiangsu, PR China
}

\begin{abstract}
Background. Ethylene response factors (ERFs) perform diverse functions in fruit development, ripening and senescence. However, the effects of postharvest treatments on ERF genes have not been widely investigated due to the lack of peach ERF genomic information. The aim of this study was to investigate the ERF genes' expression of freshly harvested peach during storage after 1-methylcyclopropene (1-MCP) treatment.

Material and methods. $10 \mu \mathrm{L} \mathrm{L}^{-1} 1-\mathrm{MCP}$ was used to fumigate peaches. Treated peaches and control peaches were stored at $20^{\circ} \mathrm{C}$ for 9 days. Fruit firmness, ethylene production and the transcript abundance of ERFs were evaluated during storage.

Results. 127 AP2/ERF genes were identified genome using RNA-sequencing (RNA-seq). Expression profiles of 39 ERF genes were considered at day 0,3,5 and 7. Results showed that 1-MCP inhibited some ERF genes' expression (e.g., Prupe.5G117800), some genes were generally up-regulated responding to 1-MCP (e.g., Prupe.6G039700), while the other ERF genes displayed no significant difference between the two groups (e.g., Prupe.1G130300).

Conclusions. These data revealed that peach ERF genes perform diverse functions during fruit growth, ripening and senescence. The different responses of ERF genes to postharvest 1-MCP treatment may be useful to understand the roles of ethylene and ERF genes in controlling technological aspects of postharvest peach conservation.
\end{abstract}

Keywords: fruit, storage, postharvest treatment, ERF, gene expression, RNA-seq

\section{INTRODUCTION}

Peach [Prunus persica (L.) Batsch] is a typical climacteric fruit due to the burst in ethylene and respiration after harvest. As an ethylene action inhibitor, 1-methylcyclopropene (1-MCP) is considered to inhibit ethylene-dependent responses and was widely studied over the past few years on account of high efficiency at relatively low concentrations and short treatment periods in various fruit and vegetables 
(Blankenship and Dole, 2003). Researches about the application of 1-MCP on peaches have been widely studied. The results of these studies indicated that ethylene production was restrained and fruit softening was delayed when fruit were stored at room temperature (Li et al., 2012; Mario-González et al., 2019), as well as at early and late harvest stage (Liguori et al., 2004).

Ethylene is a very important plant hormone and plays significant roles in plant growth, development and senescence as well as adaptation to biotic and nonbiotic stresses (Bleecker and Kende, 2000; Schaller, 2012; Ueda and Kusaba, 2015). To explore how ethylene regulates these physiological processes, genes related with ethylene biosynthesis and signal transduction have been widely studied. Research on 'Conference' pears found that 1-MCP promoted PCETR1 and repressed $P C A C S 1, P C A C O 1$ gene expression and inhibited ACO activity during cold storage (Chiriboga et al., 2013). In peach fruit, one ETR-like gene and five AP2/ERF genes were proven to be associated with ethylene production and are responsive to postharvest 1-MCP treatment (Wang et al., 2017). Since there have been lots of studies on ethylene biosynthesis and the signal transduction process, people now put more efforts into the study of ERFs, which could provide more insights into fruit ripening regulation.

The ERF genes family is a large transcription factors family and belongs to the AP2/ERF superfamily because its proteins contain a single AP2/ERF domain that is composed of about 60 to 70 amino acids involved in DNA binding (Xiu-lan et al., 2016). According to the different conserved amino acids of the AP2/ ERF domain, the ERF family can be further divided into two major subfamilies: the ERF subfamily and the CBF/DREB subfamily (Sakuma et al., 2002). As a kind of regulatory element, ERF genes usually bind GCC box (conservative sequence is AGCCGCC), participating in ethylene signal pathway, hypoxia stress reaction, pathogen stress reaction, injury reaction and other processes (Ohmetakagi and Shinshi, 1995).

The role of ERF in fruit was very limited. Overexpressing S1-ERF. B3-SRDX through transgenic tomato resulted in more ethylene production and faster softening compared with the control fruit (Liu et al., 2013). In apples, cold stress significantly induced the expression of 15 ERF genes, indicating that these ERF genes are related to cold acclimation (Zhao et al.,
2020). Similar results were also found in cold-tolerant variety-Dingjiaba Liguangtao peaches, showing that the transcript abundance of 21 transcription factors in ERF family was significantly up-regulated by cold stress (Niu et al., 2020). In addition, ERF genes related with fruit ripening were also investigated in apple (Inohara et al., 2010) and banana (Xiao et al., 2013). However, the expression patterns of ERF genes in response to postharvest treatment were not clear in peach.

The regulation of 1-MCP on the expression of ERFs is very limited in peach fruit due to the lack of peach ERF genomic information. The high throughput technologies, represented by transcriptome technologies (RNA-seq) have developed rapidly in recent years, are a powerful tool for studying genes' expression in cells. To unravel the regulation mechanisms of 1-MCP treatment on peaches and also to provide a theoretical basis for molecular breeding to cultivate storable peach fruits, RNA-seq was used to determine the ERFs expression in peach fruit after postharvest 1-MCP treatment. This may provide further insights into the regulation of the ERF genes in peach ripening and senescence after postharvest 1-MCP treatment.

\section{MATERIALS AND METHODS}

\section{Plant materials and treatments and sampling}

'Xiahui 6' (Prunus persica L.) peach is a local cultivar in Jiangsu Province. Commercially mature peaches (110 days after full bloom; average flesh firmness: $16 \mathrm{~N}$ ) were harvested from an orchard in Changzhou, Jiangsu Province, China. Fruit with uniform size and without visible defects were selected for the experiment. For 1-MCP treatment, the fruit were fumigated with $10 \mu \mathrm{L} \mathrm{L}^{-1} 1$-MCP (Sinopharm Chemical Reagent Beijing Co., Ltd, China) vapor in sealed plastic boxes at room temperature for $12 \mathrm{~h}$ (Zhang et al., 2012). After treatment, the control and treated peaches were stored at $20 \pm 1^{\circ} \mathrm{C}$ with $85-90 \%$ relative humidity for 9 days. Ten fruit for each replicate with 3 biological replicates (30 fruit) for 1-MCP-treated and control group at each sampled point $(0,1,3,5,7,9)$ were used for experiment.

\section{Evaluations of fruit ripening}

Fruit firmness was determined using a hand-held Texture Analyzer (TA.XT. Plus, America) at the opposite 
sides on the equator of three replicates of 10 fruit for one replicate and the results were expressed in newtons $(\mathrm{N})$.

Fifteen fruit were chosen from thirty fruit and constituted of three replicates. Five fruit of each replicate were put into a $3.18 \mathrm{~L}$ glass jar and covered with a rubber cap. After sealing for $1 \mathrm{~h}$ at $20^{\circ} \mathrm{C}, 1 \mathrm{~mL}$ of headspace gas was inhaled into a syringe from each jar to a gas chromatograph (Agilent Technologies 7890A) fitted with flame ionization detector (FID) and a HP$\mathrm{AL} / \mathrm{S}$ column $(30 \mathrm{~m} \times 0.53 \mathrm{~mm} \times 15 \mathrm{~mm}$, Agilent, USA). The temperature of oven, inlet and detectors were 100 , 120 and $180^{\circ} \mathrm{C}$, respectively. Ethylene production was evaluated according to our previous report (Cai et al., 2018). The results were expressed as nmol kg-1 $\mathrm{s}^{-1}$.

\section{RNA extraction and RNA-seq}

Total RNA was extracted with MiniBEST Plant RNA Extraction Kit (TaKaRa Bio Inc., Japan) from frozen peach pulp tissue based on a previous report (Cai et al., 2018). RNA-seq was conducted by BGI Tech Co. Ltd. (Shenzhen, China) on an Illumina HiSeq Xten platform. Peach genome was chosen as a reference genome at GDR database (http://www. rosaceae.org/ peach/genome). Gene expression was calculated using RNA-seq by Expectation Maximization (RSEM) (Li and Dewey, 2011) and normalized using fragments per kilobase per million mapped fragments (FPKM). Based on the changes of ethylene production during the whole storage, a total of 14 samples which were sampled at day $0,3,5,7$ of the two groups were chosen for RNA-seq. One hundred and twenty-seven AP2/ERF genes were identified in the peach genome by aligning the open reading frame (ORF) to the transcription factor protein domain (data from PlntfDB). To identify the ERF genes in peach, excluding the extremely low-expressed genes, 48 genes with FPKM $>1$ were chosen to build a phylogenetic tree by using 122 ERF protein sequences of Arabidopsis as query sequences.

\section{Statistical analysis}

The data were processed by the analysis of T-test with $P$ value $<0.05\left(^{*}\right)$ using the SPSS 18.0 software (SPSS Inc., Chicago, IL, USA). Data were plotted as means $\pm \mathrm{SD}$ in figures and the figures were made using Origin Pro 7.5 G (Microcal Software, Northampton, MA).

\section{RESULTS}

\section{Peach ripening evaluation}

A continuous decreasing trend was found in peach firmness both in control and 1-MCP groups, but 1-MCP treatment delayed the decline of firmness and maintained higher fruit firmness significantly before day 9 compared with the control (Fig. 1A). Ethylene production was remarkably restrained by $1-\mathrm{MCP}$, as indicated by postponed peaks for two days and lower peak values. Lower ethylene production except for days 1 and 9 was found in 1-MCP treated peaches (Fig. 1B).

\section{Peach ERF analysis}

As shown in Figure 2, the genes were divided into 13 groups which can be distinguished through different colors. Except for the cluster marked in gray, the other clusters were in accordance with the 12 groups reported by Nakano et al. (2006), namely, groups I to $\mathrm{X}, \mathrm{VI}-\mathrm{L}$ and $\mathrm{Xb}-\mathrm{L}$.

\section{Expression of peach ERF genes in response to 1-MCP treatment}

The 39 ERF genes were clustered with AtERFs (ERFs in Arabidopsis) in different groups except for the 9 genes marked in gray which didn't cluster in any group (Fig. 1). The heatmap was made using $39 \mathrm{ERF}$ genes transcripts, indicating differential expression of peach ERF genes (Fig. 3). The genes' expression could be roughly divided into two independent clusters. Genes in the first cluster were highly expressed at day 0 followed by observably lower expression with the extending of shelf life. The other genes showed an increasing pattern overall, and transcript level was inhibited by $1-\mathrm{MCP}$ treatment. The expression of the 39 ERF genes was showed in Figures 4, 5, 6 and 7.

As shown in Figure 4, four ERF genes were clustered into group I and group II respectively based on the phylogenetic tree. The eight genes showed different expression patterns during $20^{\circ} \mathrm{C}$ storage. Some genes' transcript level was significantly inhibited by 1-MCP treatment before day 7 (Prupe.7G194400; Prupe.3G157100; Prupe.5G117800), while 1-MCP also promoted some genes' expression compared with the control (Prupe.6G182200; Prupe.1G513600; Prupe.1G432000) at day 3 and 5. Six genes were 
Cai, H., Han, S., Wang, H., Yu, M., Ma, R., Yu, Z. (2021). The regulation of 1-methylcyclopropene treatment on the subfamily genes expression of ethylene response factors in peaches during storage. Acta Sci. Pol. Technol. Aliment., 20(3), 313-323. http://dx.doi. org/10.17306/J.AFS.2021.0943
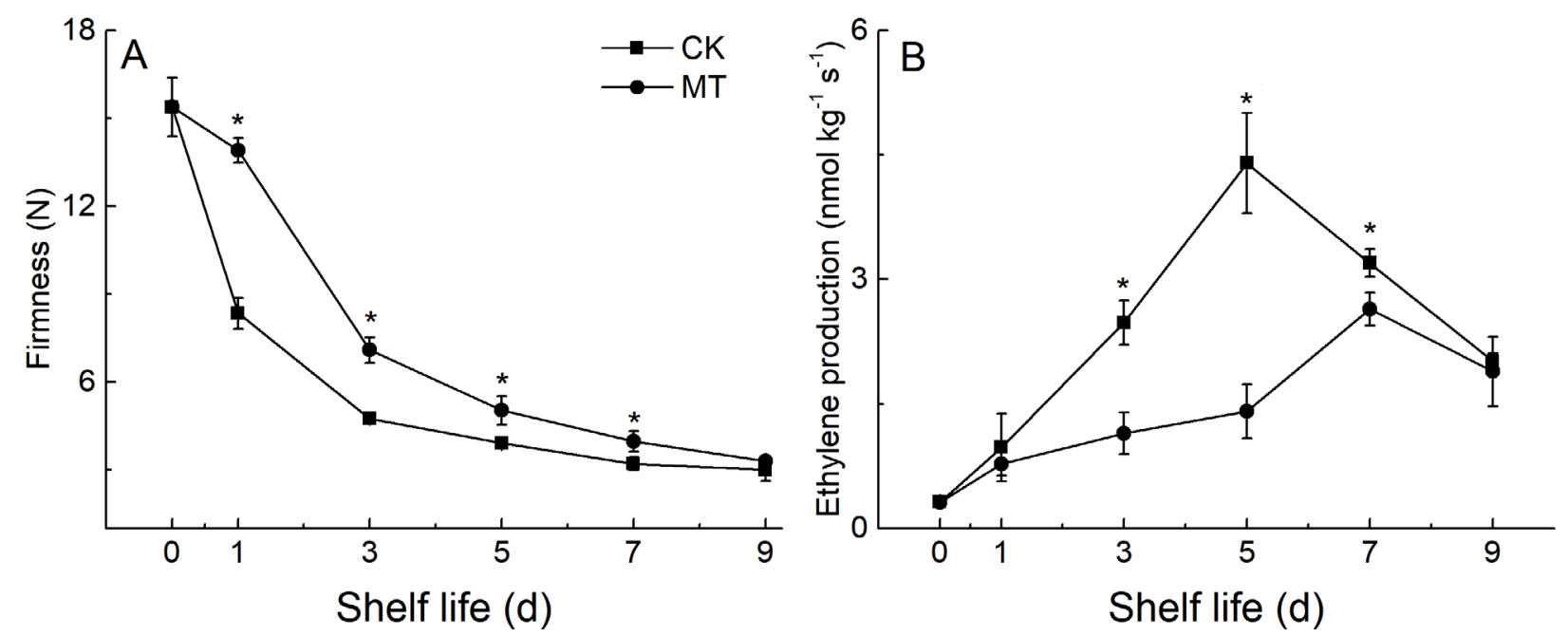

Fig. 1. Effect of 1-MCP on peach fruit firmness $(\mathrm{A}, \mathrm{N})$ and ethylene production $\left(\mathrm{B}, \mathrm{nmol} \mathrm{kg} \mathrm{s}^{-1} \mathrm{~s}^{-1}\right)$ during storage at $20^{\circ} \mathrm{C}$. $\mathrm{CK}-$ control group, MT $-1-\mathrm{MCP}$ treatment group. All the values are expressed as means $\pm \mathrm{SD}$ of three replicates. Asterisks denote significant difference $(P<0.05)$ under T-test between the 1-MCP-treated and control group at same sampled point, no asterisk means no significant difference between the two groups

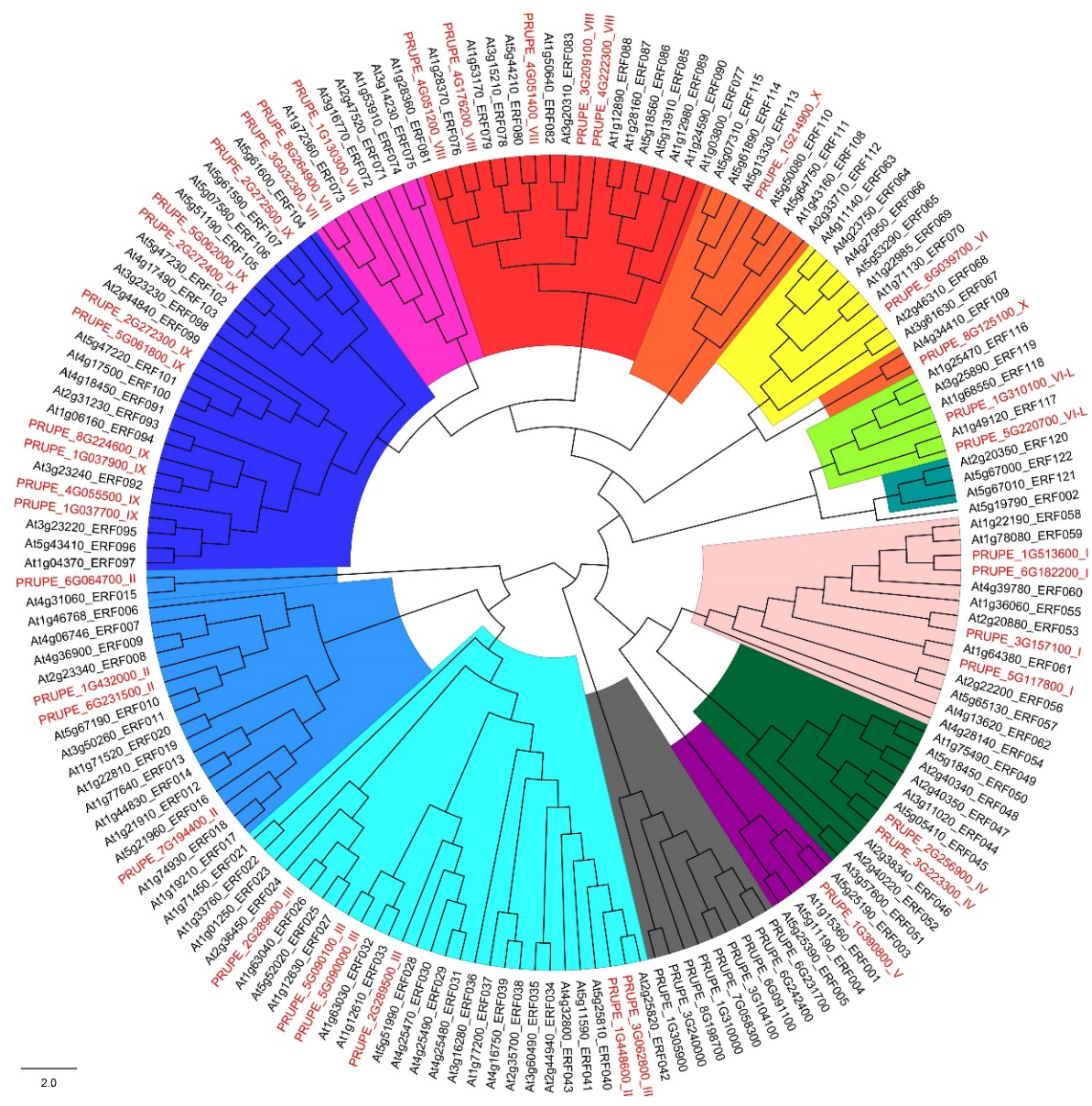

Fig. 2. Phylogenetic analysis of peach ERF genes. The ERF protein sequences of Arabidopsis thaliana were obtained from TAIR. A phylogenetic tree was constructed using the neighbour-joining method (NJ) and analysed using the Poisson correction models with 1000 bootstrap replicates in MEGA6.0 then processed by FigTree 
Cai, H., Han, S., Wang, H., Yu, M., Ma, R., Yu, Z. (2021). The regulation of 1-methylcyclopropene treatment on the subfamily genes expression of ethylene response factors in peaches during storage. Acta Sci. Pol. Technol. Aliment., 20(3), 313-323. http://dx.doi. org/10.17306/J.AFS.2021.0943

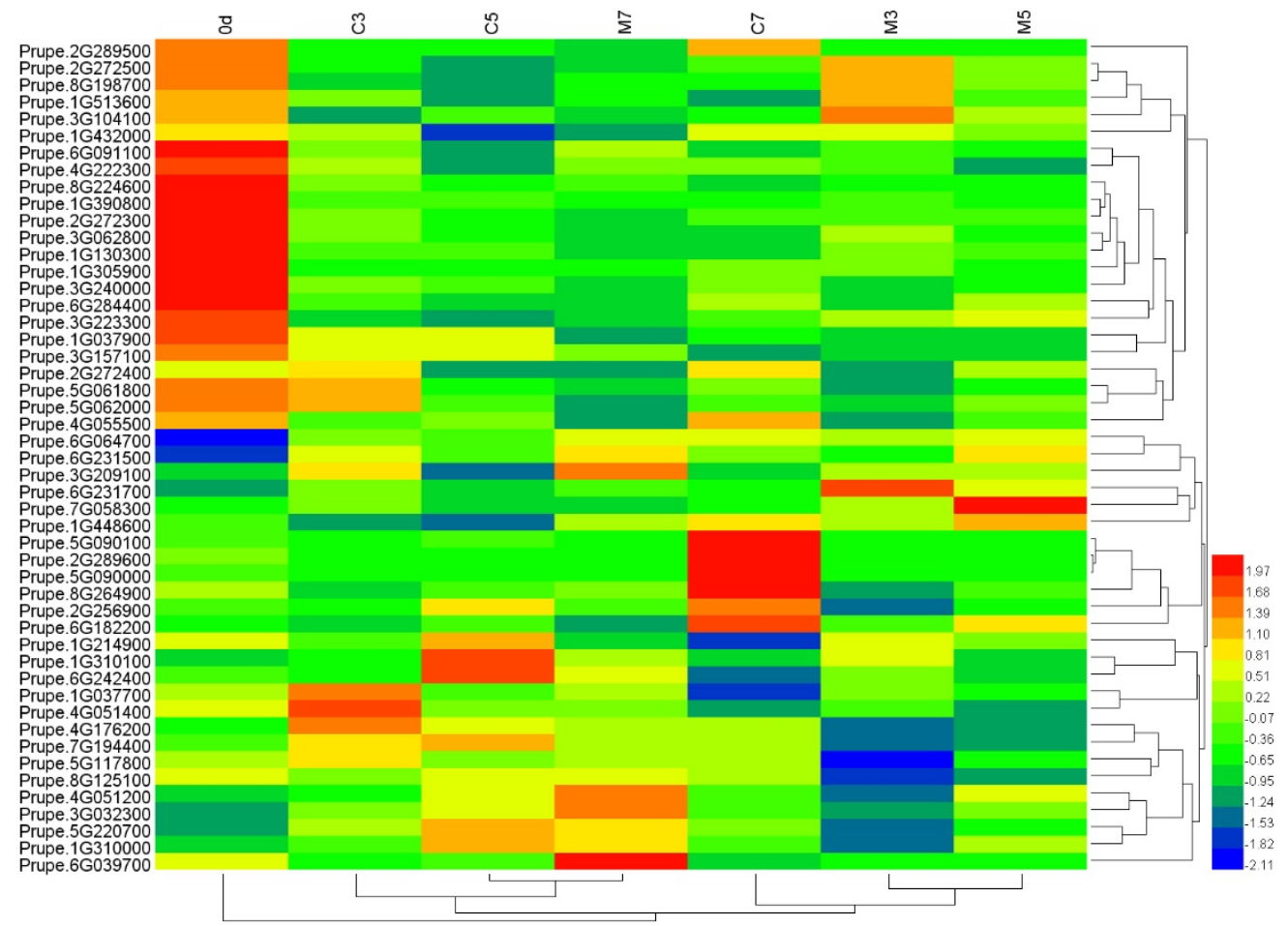

Fig. 3. Hierarchical cluster analysis of ERF genes expression during storage in peach fruit. $\mathrm{C} 3,5,7$ represents control group at day 3,5, 7; M3, 5, 7 represents 1-MCP treatment group at day 3, 5, 7. The figure was made with Heatmap Illustrator (HemI, 1.0.0.3). The data were the means of two replicates and were processed with Z-score normalization and hierarchically clustered using Spearman distance

Fig. 4. The expression of ERF genes in group I (A - four pictures on the left) and II (B-four pictures on the right) between control and 1-MCP treatment group. FPKM - fragments per kilobase of exon model per million mapped fragments of each gene, $\mathrm{CK}$ - control group, MT 1-MCP treatment group. Error bars on each column represent SDs from 2 biological replicates. Asterisks denote significant difference $(P<0.05)$ under T-test between the 1-MCP-treated and control group at same sampled point, no asterisk means no significant difference between the two groups
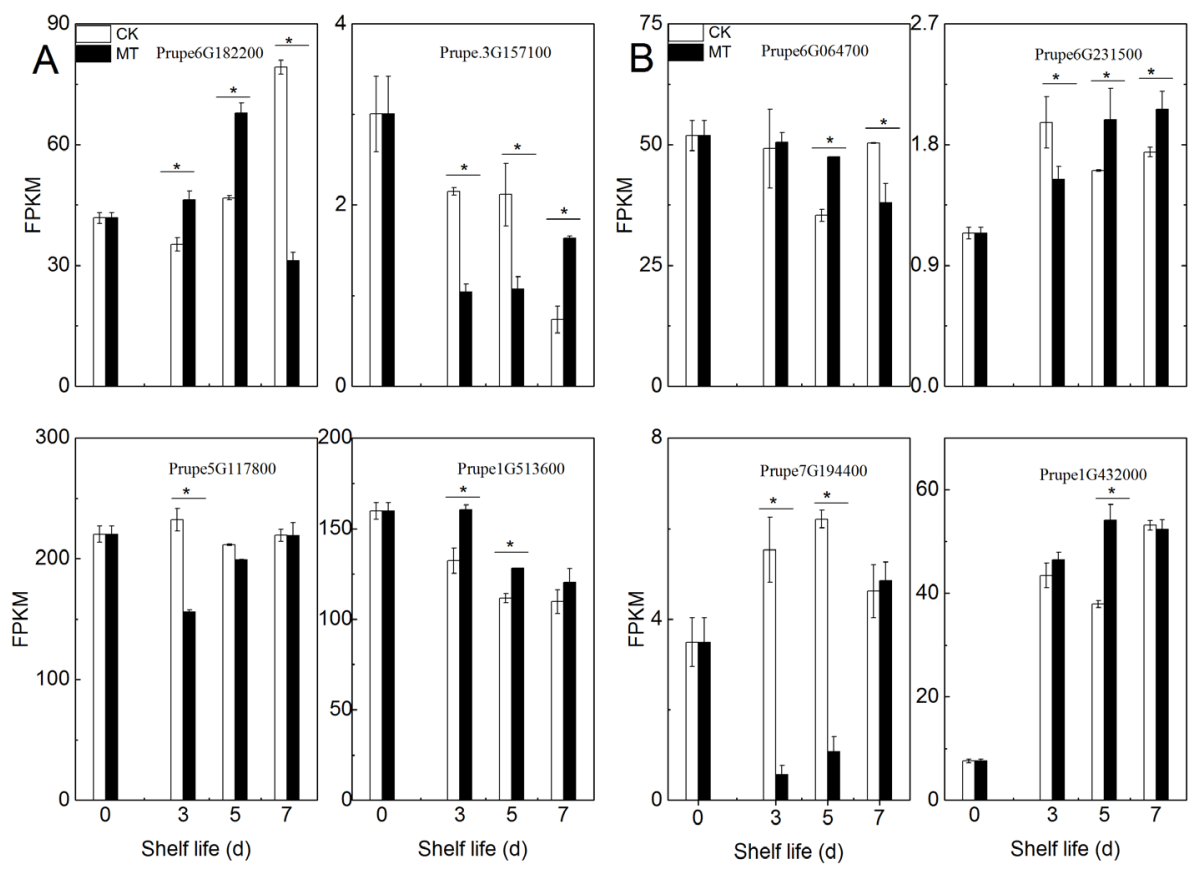

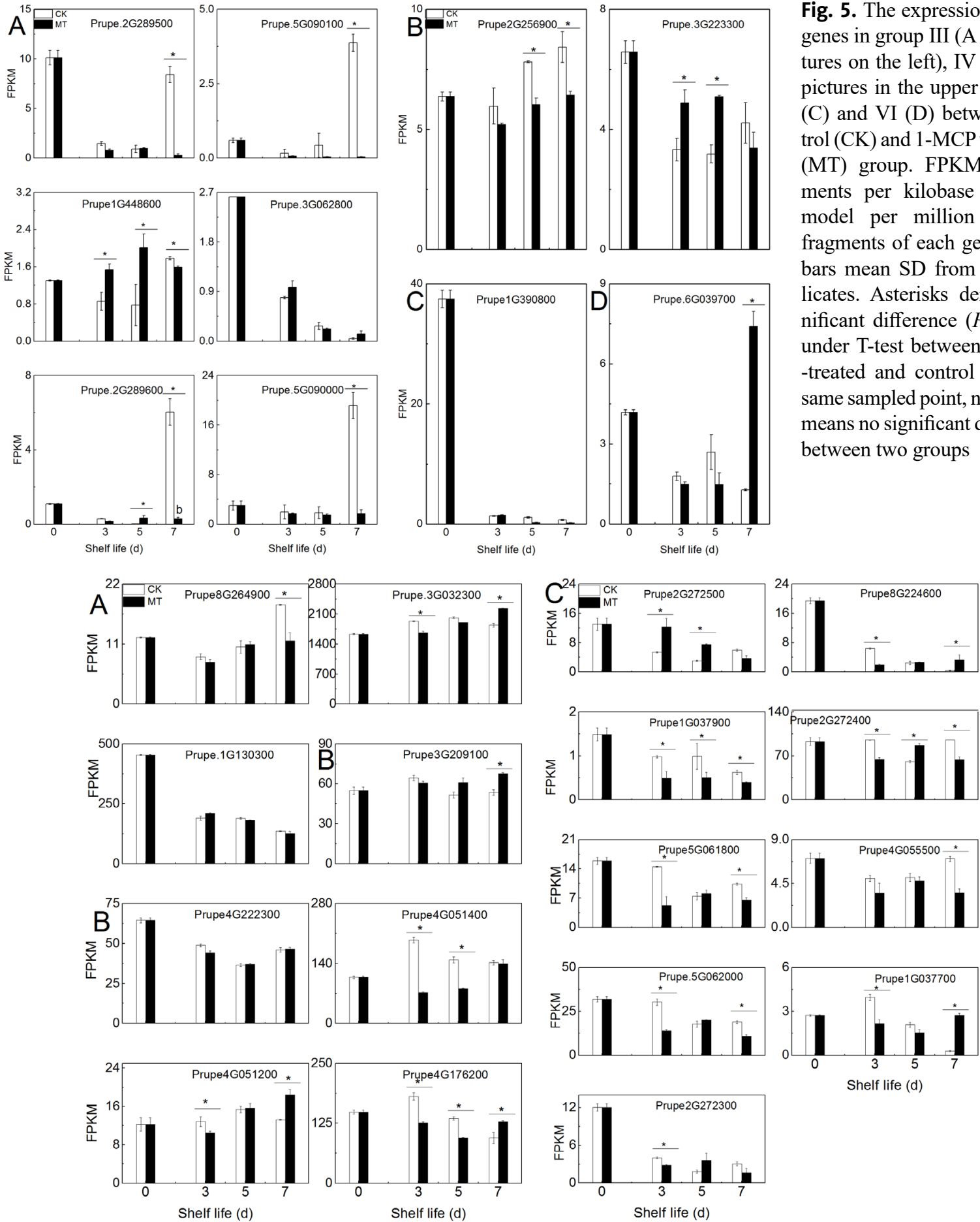

Fig. 6. The expression of ERF genes in group VII (A - first three pictures at the top left), VIII (B - five pictures at the bottom left $)$ and IX $(\mathrm{C}$ - nine pictures on the right) between control $(\mathrm{CK})$ and 1-MCP treatment (MT) group. FPKM - fragments per kilobase of exon model per million mapped fragments of each gene. Error bars on each column represent SDs from 2 biological replicates. Asterisks denote significant difference $(P<0.05)$ under T-test between 1-MCP-treated and control group at same sampled point, no asterisk means no significant difference between two groups 
Fig. 7. The expression of ERF genes in group X (A - two pictures above) and VI-L (B - two pictures below) between control (CK) and 1-MCP treatment (MT) group. FPKM - fragments per kilobase of exon model per million mapped fragments of each gene. Error bars mean SD from two replicates. Asterisks denote significant difference $(P<0.05)$ under T-test between 1-MCP-treated and control group at same sampled point, no asterisk means no significant difference between two groups
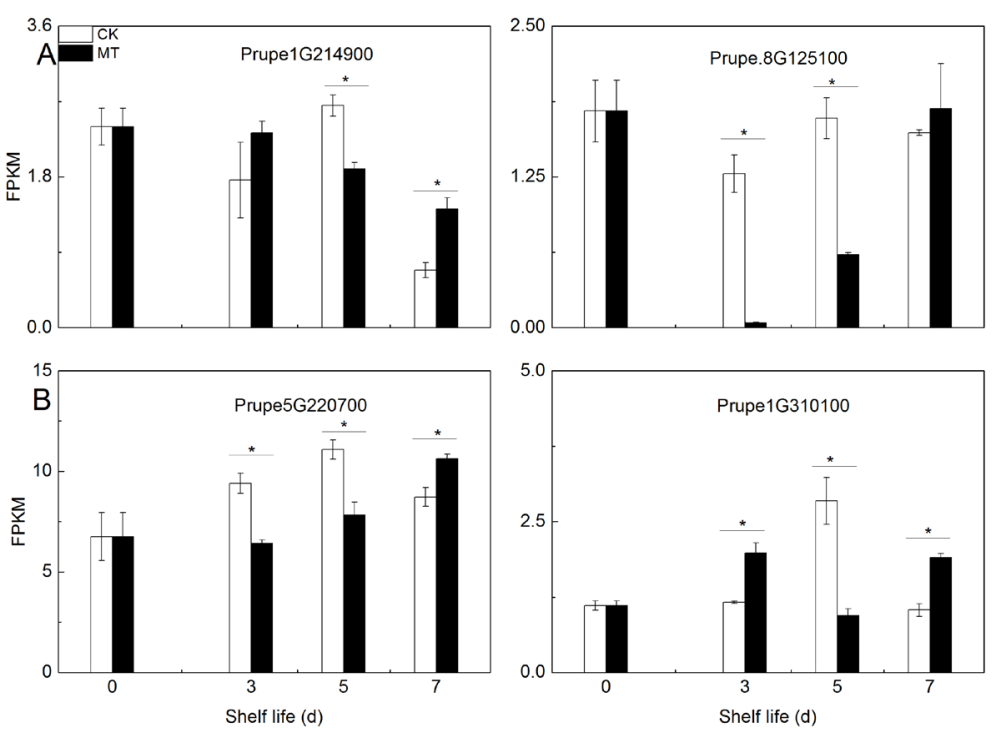

clustered with AtERFs at group III (Fig. 5A), transcript abundance of five genes reduced after harvest (day 0 ) in two groups, and 1-MCP treatment extremely inhibited their expression at day 7 . There were two genes that were clustered into group IV and showed opposite change patterns (Fig. 5B), 1-MCP treatment downregulated the transcript level of Prupe.2G256900, while an opposite, up-regulating level was found in the 1-MCP-treated group in Prupe.3G223300 although its expression decreased during $20^{\circ} \mathrm{C}$ storage. Only one gene was clustered into group V (Fig. 5C) and its expression was extremely low during storage.

Prupe.6G039700 was in group VI and showed the similar change pattern with ethylene production that its level was restrained by $1-\mathrm{MCP}$ before day 5 . Three, five and nine genes varied with transcripts abundance were clustered into group VII, VIII and IX respectively (Fig. 6A, 6B, 6C). 1-MCP treatment significantly suppressed the expression of Prupe.3G032300, Prupe.4G051200, Prupe.8G224600, Prupe.2G272400, Prupe.5G061800, 5G0620000 and Prupe.1G037700 at day 3 , inhibited the transcript level of Prupe.4G051400 and Prupe.4G176200 at day 3 and 5, and also decreased the level of Prupe.8G264900, Prupe.2G272400, Prupe.4G055500 and 5G0620000 at day 7. The expression of Prupe.1G037900 was totally suppressed by 1-MCP treatment during the whole storage. While some genes' mRNA level was enhanced in response to 1-MCP treatment at day 7 (Prupe.3G032300; Prupe.3G209100; Prupe.4G051200; Prupe.4G176200; Prupe.8G224600; Prupe.1G037700). 1-MCP treatment also increased the expression of Prupe.2G272500 at day 3 and 5. There also have some genes that showed no significant difference between the two groups (Prupe.1G130300; Prupe.4G222300). Only two genes were clustered into group $\mathrm{X}, 1-\mathrm{MCP}$ treatment extremely inhibited the expression of Prupe.8G125100 (more than 2-fold) at day 3 and 5. Another gene, Prupe.1G214900, showed lower expression level in 1-MCP group at day 5 and higher level at day 7 (Fig. 7A).

According to the ERFs in Arabidopsis, two genes were assigned to VI-L group including Prupe.5G220700 and Prupe.1G310100 (Fig. 7B). The expression pattern of Prupe.5G220700 was in accordance with ethylene production. 1-MCP inhibited its level and postponed the peak value for at least two days. Prupe.1G310100 in peaches treated with 1-MCP fluctuated during the whole storage, accompanied by a significantly higher level during storage except for day 5, during which a sudden increase in Prupe.1G310100 level appeared in the control group. During storage at room temperature, 
these genes showed different change patterns, indicating that peach ERF genes members performed diverse functions during fruit ripening and senescence.

\section{DISCUSSION}

Recently, studies about ERF genes have gained more and more attention. Studies have shown that ERF genes perform various functions in plant development and growth, including seed germination, root initiation, leaf emergence, floral development, fruit ripening, and organ senescence (Karlova et al., 2014; Licausi et al., 2013). Besides, ERF genes also play important roles in plant defense such as wheat TaERF3 involvement in adaptation to drought (Rong et al., 2014), three members of DREB family (DREB1A/ CBF3, DREB1B/CBF1, DREB1C/CBF2) induced by cold stress in Arabidopsis (Mizoi et al., 2012).

In fruit, some studies focused on the ERF genes related with fruit colour, texture, flavor and ripening by using transgenosis or RNAi mostly existing in model fruit Tomato LeERF1 was found to be associated with fruit ripening and softening ( $\mathrm{Li}$ et al., 2007) and $\mathrm{Le}$ $E R F 2$ could trigger fruit ethylene synthesis (Zhang et al., 2009). PbERF22 in pears was found to respond to jasmonate, ethylene signals and affect fruit anthocyanin biosynthesis (Wu et al., 2020). ERF genes have not been widely studied in different fruit species for the lack of genomic information.

1-MCP treatment has proven to be effective in prolonging the postharvest life of fresh fruit, especially climacteric fruit. Here, 1-MCP treatment retarded the decline of fruit firmness, maintained higher fruit SSC and TA, reduced respiration rate and exogenous ethylene production thus delaying fruit ripening. These findings were in accordance with our previous studies (Cai et al., 2018; Wu et al., 2018). Since 1-MCP is an ethylene inhibitor, ethylene is an important regulator during fruit development, ripening and senescence, and largely acts through families of transcription factors. However, comprehensive analysis about the transcriptional responses of ERF genes to various postharvest treatments on fruit was lacking. To better understand how 1-MCP delayed fruit ripening by inhibiting ethylene production, the ERF genes expression profile in response to $1-\mathrm{MCP}$ treatment was taken into consideration.
In the present study, $127 \mathrm{AP} / \mathrm{ERF}$ genes were identified from the peach genome by using RNA-seq tech. Among these genes, $39 \mathrm{ERF}$ genes distributed in different 12 groups with FPKM $>1$ were identified based on phylogenetic tree by using 122 AtERF genes' protein sequence as alignment sequence (Fig. 1). According to the previous studies, the 12 groups can be further divided into ERF subfamily (group B) and CBF/DREB subfamily (group A). Of the 12 groups, group I, II, III and IV belong to group A, and group B includes group V, VI, VII, VIII, IX and group X (Nakano et al., 2006; Sakuma et al., 2002). ERF genes in different groups perform different functions. $\mathrm{CBF} /$ DREB genes are often related with ABA, drought-, and cold-responsive genes and genes in the ERF subfamily respond to ethylene, pathogens, and wounding.

Here, phylogenetic analysis showed that Prupe.3G1571100 and Prupe.5G117800 were highly homologous to AtERF53 (Fig. 1), which regulates droughtresponsive gene expressions (TAIR: https://www. arabidopsis.org/). However, 1-MCP down-regulated their expression at day 3 and 5, suggesting that the regulation of AtERF53 on drought may be related to ethylene. In the phylogenetic tree, the other two genes in group I showed a slightly higher transcript abundance in the 1-MCP-treated group, and are closer to AtERF59 which play roles in mediating light and ethylene signaling (TAIR: https://www.arabidopsis.org/), indicating that these two genes in peach may be negatively regulated by ethylene. In group II, an interesting phenomenon was found: 1-MCP significantly inhibited the transcript level of Prupe.7G194400 (over 4-fold at day 3 and 5) which shared high homology with AtERF18. In the TAIR database (TAIR: https://www. arabidopsis.org/), AtERF18 was described as a participant in defense responses towards insects, wounding and vasculature development. We may deduce that these bioprocess in plants are positively regulated by ethylene.

In peaches, Wang et al. (2019a) found that 1-MCP greatly inhibited the increase of ABA content by suppressing the expression of PpERF3 (Prupe.7G194400), which is consistent with our results. Among the six ERF genes in group III, Prupe.2G289500 was clustered into a clade with AtERF28, which belongs to the CBF4/DREB1D subfamily and was thought to be involved in the response to drought stress and abscisic 
acid treatment, but not to low temperature (TAIR: https://www.arabidopsis.org/). However, the expression of Prupe.2G289500 dropped sharply after harvest and recovered in control group at day 7 but not in the 1-MCP-treated group, indicating the inhibition action of this gene on peach ripening. In peaches, PpeERF2 was classified in group III and it was proven to be a fruit ripening inhibitor for repressing the transcription level of PpeNCED2, PpeNCED3 and PpePG1, the three genes are ripening related genes (Wang et al., 2019b). In Figure 1, Prupe.5G090100 and Prupe.5G090000 shared high homology with AtERF32 and AtERF33, which were described as negatively regulating genes' expression related with gibberellic acid (GA) biosynthesis (TAIR: https://www.arabidopsis.org/). 1-MCP inhibited the two ERF genes' transcript level especially at day 7 , which matches the reports that 1-MCP could up-regulate the genes' expression of GA biosynthesis (Shinozaki et al., 2015). Two ERF genes in group IV were closer to AtERF44 and AtERF45 in the phylogenic tree, which are activators and participate in drought stress tolerance intransgenic Arabidopsis plants (TAIR: https://www.arabidopsis.org/). However, these two genes in peach showed opposite expression profiles, suggesting that ethylene affect little on them.

Among the ERF subfamily, Prupe.1G390800 was clustered into group $\mathrm{V}$, next to AtERF1, which was considered to participate in wax biosynthesis, overexpression of AtERF1 results in glossy leaf phenotype and increased drought tolerance (TAIR: https://www. arabidopsis.org/). This gene showed lower transcript abundance in the 1-MCP group, indicating that 1-MCP has a negative regulatory effect on these processes. Prupe.6G039700 was the only ERF gene in group VI which shares high homology with AtERF67 and AtERF68 - which are up-regulated transcriptionally by cytokinin (TAIR: https://www.arabidopsis.org/). Here, the mRNA level of this gene was enhanced by 1-MCP (about 4.8-fold) at day 7, showing that the role of ethylene and cytokinin is antagonistic. Three ERF genes in group VII were closer to AtERF73, which was related to hypoxia responsive and low oxygen signaling (TAIR: https://www.arabidopsis.org/). However, the mRNA abundance of three genes in 1-MCP and control group have no obvious difference, so we may deduce that the above physiological processes are very slightly affected by ethylene. In group VIII,
Prupe.4G176200 was clustered into a clade with AtERF76 which was thought to be a repressor and involved in MeJA and fungal responsive (TAIR: https:// www.arabidopsis.org/), however, Prupe.4G176200 was inhibited by 1-MCP at day 3 and 5. Similar results were also found in Prupe.4G051200, indicating that the two ERF genes have a positive connection with fruit ripening. Nine genes were assigned to group IX but not all genes had response to 1-MCP. According to phylogenetic analysis, Prupe.1G037700 shared high homology with AtERF95, which participated in salt responsiveness in plants (TAIR: https://www.arabidopsis.org/) and 1-MCP down-regulated this gene's expression on day 3 , showing that salt responsiveness in plants may need the participation of ethylene.

Prupe. 1G037900 was in a small clade with AtERF92, which was an activator and was involved in an ethylene signaling cascade in the downstream of EIN2 and EIN3 (TAIR: https://www.arabidopsis.org/). Our results fit well with AtERF92 that Prupe.1G037900 was inhibited by $1-\mathrm{MCP}$ in $20^{\circ} \mathrm{C}$ storage, suggesting that this gene has a positive relation with peach ripening. Similar 1-MCP inhibition effects were also found in Prupe.4G055500, Prupe.5G061800, Prupe.5G062000 and Prupe.2G272400, showing that these ERF genes in the CBF/DREB subfamily respond to ethylene. In group X, Prupe.8G125100 shared high homology with AtERF109, which was involved in the defense and regulation of the auxin mediated signaling pathway and tryptophan metabolic process (TAIR: https:// www.arabidopsis.org/). Here, 1-MCP down-regulated this gene's expression by about 3-fold on days 3 and 5 , suggesting that these processes need the participation of ethylene. Two ERF genes in group VI-L were clustered with AtERF117, while there was no specific function for this gene. According to the trends, we may deduce that the two genes are regulated by ethylene.

\section{CONCLUSION}

In general, ERF genes were identified, and expression profiles were measured in response to postharvest 1-MCP treatment to better understand the regulation of 1-MCP and ethylene on peach ripening and senescence. 1-MCP treatment retarded fruit firmness decline, and inhibited ethylene production. 48 ERF genes with FPKM $>1$ were obtained and showed 
different performance patterns. The results indicate that peach ERF genes are involved in regulating various bioprocesses through changes in the expression of key genes. Further analysis is required in order to identify the gene targets for specific physiological and biochemical processes.

\section{REFERENCE}

Blankenship, S. M., Dole, J. M. (2003). 1-Methylcyclopropene: a review. Postharv. Biol. Technol., 28, 1-25.

Bleecker, A. B., Kende, H. (2000). Ethylene: a gaseous signal molecule in plants. Ann. Rev. Cell Develop. Biol., $16,1-18$.

Cai, H., An, X., Han, S., Jiang, L., Yu, M., Ma, R., Yu, Z. (2018). Effect of 1-MCP on the production of volatiles and biosynthesis-related gene expression in peach fruit during cold storage. Postharv. Biol. Technol., 141, 50-57.

Chiriboga, M. A., Saladié, M., Bordonaba, J. G., Recasens, I., Garciamas, J., Larrigaudière, C. (2013). Effect of cold storage and 1-MCP treatment on ethylene perception, signalling and synthesis: Influence on the development of the evergreen behaviour in 'Conference' pears. Postharv. Biol. Technol., 86, 212-220.

Inohara, H., Honjo, Y., Yoshii, T., Akahani, S., Hattori, K., Okamoto, S., ..., Kubo, T. (2010). The role of ethylene and cold temperature in the regulation of the apple POLYGALACTURONASE1 gene and fruit softening. Plant Physiol., 153, 294-305.

Karlova, R., Chapman, N., David, K., Angenent, G. C., Seymour, G. B., De Maagd, R. A. (2014). Transcriptional control of fleshy fruit development and ripening. J. Exp. Bot., 65, 4527-4541.

Li, B., Dewey, C. N. (2011). RSEM: accurate transcript quantification from RNA-Seq data with or without a reference genome. BMC Bioinform., 12, 323-323.

Li, Y., Zhu, B., Xu, W., Zhu, H., Chen, A., Xie, Y., ..., Luo, Y. (2007). LeERF1 positively modulated ethylene triple response on etiolated seedling, plant development and fruit ripening and softening in tomato. Plant Cell Rep., 26, 1999-2008.

Li, Z., Li, J., Yun, S., Luo, H., Kang, R., Yu, Z. (2012). Postharvest 1-methylcyclopropene and ethephon treatments differently modify protein profiles of peach fruit during ripening. Food Res. Int., 48.

Licausi, F., Ohme-Takagi, M., Perata, P. (2013). APETALA2/ Ethylene Responsive Factor (AP2/ERF) transcription factors: mediators of stress responses and developmental programs. New Phytol., 199, 639-649.
Liguori, G., Weksler, A., Zutahi, Y., Lurie, S., Kosto, I. (2004). Effect of 1-methylcyclopropene on ripening of melting flesh peaches and nectarines. Postharv. Biol. Technol., 31, 263-268.

Liu, M., Pirrello, J., Kesari, R., Mila, I., Roustan, J., Li, Z., ..., Regad, F. (2013). A dominant repressor version of the tomato Sl-ERF.B3 gene confers ethylene hypersensitivity via feedback regulation of ethylene signaling and response components. Plant J., 76, 406-419.

Mario-González, L. A., Buitrago, C. M., Balaguera-López, H. E. (2019). Effect of 1-methylcyclopropene and ethylene on the physiology of peach fruits (Prunus persica L.) cv. Dorado during storage. Rev. Colom. Cienc. Hortíc., 13, 37-45.

Mizoi, J., Shinozaki, K., Yamaguchishinozaki, K. (2012). AP2/ERF family transcription factors in plant abiotic stress responses. BBA - Gene Regul. Mech., 1819, 86-96.

Nakano, T., Suzuki, K., Fujimura, T., Shinshi, H. (2006). Genome-wide analysis of the ERF gene family in arabidopsis and rice. Plant Physiol., 140, 411.

Niu, R., Zhao, X., Wang, C., Wang, F. (2020). Transcriptome profiling of Prunus persica branches reveals candidate genes potentially involved in freezing tolerance. Scientia Hortic., 259, 108775.

Ohmetakagi, M., Shinshi, H. (1995). Ethylene-inducible DNA binding proteins that interact with an ethyleneresponsive element. Plant Cell, 7, 173-182.

Rong, W., Qi, L., Wang, A., Ye, X., Du, L., Liang, H., Xin, Z., Zhang, Z. (2014). The ERF transcription factor TaERF3 promotes tolerance to salt and drought stresses in wheat. Plant Biotechnol. J., 12, 468-479.

Sakuma, Y., Liu, Q., Dubouzet, J. G., Abe, H., Shinozaki, K., Yamaguchi-Shinozaki, K. (2002). DNA-binding specificity of the ERF/AP2 domain of arabidopsis DREBs, transcription factors involved in dehydrationand cold-inducible gene expression. Biochem. Biophys. Res. Comm., 290, 998-1009.

Schaller, G. E. (2012). Ethylene and the regulation of plant development. BMC Biol., 10, 9.

Shinozaki, Y., Hao, S., Kojima, M., Sakakibara, H., Ozeki-Iida, Y., Zheng, Y., ..., Rose, J. K. C. (2015). Ethylene suppresses tomato (Solanum lycopersicum) fruit set through modification of gibberellin metabolism. Plant J., 83, 237-251.

Ueda, H., Kusaba, M. (2015). Strigolactone regulates leaf senescence in concert with ethylene in Arabidopsis. Plant Physiol., 169, 138.

Wang, X., Ding, Y., Wang, Y., Pan, L., Niu, L., Lu, Z., ..., Wang, Z. (2017). Genes involved in ethylene signal 
transduction in peach (Prunus persica) and their expression profiles during fruit maturation. Scient. Hortic., 224, 306-316.

Wang, X., Zeng, W., Ding, Y., Wang, Y., Niu, L., Yao, J.-L., ..., Li, G. (2019a). PpERF3 positively regulates ABA biosynthesis by activating PpNCED2/3 transcription during fruit ripening in peach. Hortic. Res., 6, 1-10.

Wang, X. B., Zeng, W. F., Ding, Y. F., Wang, Y., Niu, L., Yao, J. L., ..., Wang, Z. Q. (2019b). Peach ethylene response factor PpeERF2 represses the expression of ABA biosynthesis and cell wall degradation genes during fruit ripening. Plant Sci., 283, 116-126.

Wu, T., Liu, H.-T., Zhao, G.-P., Song, J.-X., Wang, X.-L., Yang, C.-Q., ..., Xu, L.-F. (2020). Jasmonate and ethylene-regulated ethylene response factor 22 promotes lanolin-induced anthocyanin biosynthesis in 'Zaosu' pear (Pyrus bretschneideri Rehd.) fruit. Biomolecules, 10, 278.

Wu, X. Q., An, X. J., Yu, M. L., Ma, R. J., Yu, Z. F. (2018). 1-Methylcyclopropene treatment on phenolics and the antioxidant system in postharvest peach combined with the liquid chromatography/mass spectrometry technique. J. Agric. Food Chem., 66, 6364-6372.
Xiao, Y., Chen, J., Kuang, J., Shan, W., Xie, H., Jiang, Y., $\mathrm{Lu}, \mathrm{W}$. (2013). Banana ethylene response factors are involved in fruit ripening through their interactions with ethylene biosynthesis genes. J. Exp. Bot., 64, 2499-2510.

Xiu-lan, X., Xue-ren, Y., Kun-song, Ch. (2016). Roles of APETALA2/ethylene-response factors in regulation of fruit quality. Crit. Rev. Plant Sci., 35, 120-130.

Zhang, L., Jiang, L., Shi, Y., Luo, H., Kang, R., Yu, Z. (2012). Post-harvest 1-methylcyclopropene and ethephon treatments differently modify protein profiles of peach fruit during ripening. Food Res. Int., 48, 609-619.

Zhang, Z. J., Zhang, H. W., Quan, R. D., Wang, X. C., Huang, R. F. (2009). Transcriptional regulation of the ethylene response factor LeERF2 in the expression of ethylene biosynthesis genes controls ethylene production in tomato and tobacco. Plant Physiol., 150, 365-377.

Zhao, J., Quan, P., Liu, H., Li, L., Qi, S., Zhang, M., ..., Ma, B. (2020). Transcriptomic and metabolic analyses provide new insights into the apple fruit quality decline during long-term cold storage. J. Agric. Food Chem., 68(16), 4699-4716. https://doi.org/10.1021/acs.jafc.9b07107 